\title{
¿SE HAN INTRODUCIDO LAS TIC EN LA FORMACIÓN INICIAL DE DOCENTES? ESTUDIO DE CASO EN EL CENTRO DE MAGISTERIO "VIRGEN DE EUROPA"
}

\section{HAS ITC BEEN INCLUDED IN INITIAL TEACHER TRAINING? STUDY CASE IN TEACHER TRAINING CENTRE "VIRGEN DE EUROPA"}

\author{
Claudia I. Ruiz López \\ Centro de Magisterio "Virgen de Europa" \\ Adscrito a Universidad de Cádiz
}

Fecha de recepción: 05/02/2015

Fecha de aceptación: 19/04/2015

\section{RESUMEN}

Esta investigación se desarrolla en el marco del estudio de casos de un centro universitario de formación inicial del profesorado: el Centro de Magisterio "Virgen de Europa", adscrito a la Universidad de Cádiz (situado en La Línea de la Concepción). Ante la difusión de las tecnologías y las posibilidades que ofrecen en el marco de la educación, analizar la realidad circundante y extraer conclusiones sobre la misma se revela como interesante para reflexionar sobre la praxis y optimizar los procesos de enseñanza a futuros profesores. Partimos de una visión optimista que considera que la incorporación es más efectiva y real de lo que pudiera desprenderse de los estudios más científicos que cuestionan la capacidad de adaptación del profesorado a las demandas sociales.

En este sentido, hemos entrevistado a profesores universitarios y desarrollado un cuestionario que se ha administrado al alumnado universitario en dos fases (curso 20082009 y 2012-1013) y que trata de comprobar en qué medida se incorporan las tecnologías a su trayectoria formativa (partiendo del bachillerato y pasando por su formación inicial como docentes) acercándonos a la previsión de uso futuro de las tecnologías que tienen. Nos centraremos en la percepción de la incorporación en su paso por el centro de magisterio.

Paralelamente, en la primera fase, diseñamos y administramos un cuestionario al profesorado universitario. Esta información cuantitativa se completa con las entrevistas realizadas al profesorado en la segunda fase del estudio.

Palabras clave: Métodos para la formación inicial del profesorado, tecnologías de la educación, estudio de casos, influencia del uso de TIC.

\section{ABSTRACT}

This research is developed within a case study framework in a Centre of Initial Education and Teacher Training (private school "Virgen de Europa", Cádiz University, Spain, located in La Línea de la Concepción). In today's ITC environments in education, we consider interesting to analyse the possibilities that technologies offer, precisely to reflect on our university teaching practices and improve future teacher skills. We start with an optimistic vision that considers the addition is more effective and real than it might show more scientific studies that question the adaptability of teachers to social demands.

In this sense, we have interviewed university professors and elaborated a questionnaire for our students. The questionnaire has been distributed in two phases: first, in the 
¿Se han introducido las tic en la formación inicial de docentes? estudio de caso en el centro de magisterio "Virgen de Europa"

academic year 2008-2009 and in a second occasion in 2012-2013, just to analyse how technologies were incorporated in a first and a second moment at this centre. In addition, we try to find out if our students are thinking about using ITC in their future professional performance. Similarly, we developed and distributed as well a questionnaire for university professors in the first phase. These quantitative data are completed with qualitative evidence from interviews addressed to university teachers.

Keywords: Training methods, technology uses in education, case studies, influence of technology

\section{INTRODUCCIÓN}

Como producto del estudio de I+D Políticas educativas autonómicas y sus efectos sobre la innovación pedagógica en el uso de las TIC en los centros escolares (aprobado dentro del Plan Nacional de I+D, 2004-2007 y dirigido por D. Juan de Pablos Pons) nace en nosotros el interés por profundizar en el rol desempeñado por el docente que incorporaba las TIC en su dinámica de trabajo, concretamente en qué competencias debía poner en juego para desempeñar buenas prácticas educativas basadas en el uso de las tecnologías.

Pese a que no se apreciaba una diferencia sustancial en el modo de organización escolar del momento respecto a épocas anteriores, los docentes descritos por los CEP de las zonas estudiadas (zonas de la provincia de Cádiz y Sevilla) compartieron con nosotros su percepción del proceso de incorporación de las tecnologías en un momento en el que, a juzgar por la dificultad para identificar las que se consideraron buenas prácticas en el uso de TIC por parte de los mencionados CEP, aún no estaban muy instauradas. El perfil del profesorado era particular: sus inquietudes en torno a la educación y la innovación eran evidentes.

Indudablemente, las tecnologías irrumpían en las aulas e implicaban un nuevo docente (que se traducía o provenía, en una relación circular, de otro perfil de alumnado). Se vislumbraban desde los sectores prácticos que era necesario enfrentar posiciones más tradicionales en el desarrollo de las clases con planteamientos más constructivos del aprendizaje que incorporaran como recursos las tecnologías de la información y la comunicación.

En este marco, podíamos encontrar detractores del uso y partidarios: ¿era posible ser detractor? ¿podían no incluirse las tic en la práctica? ¿cuál era el motivo de la resistencia?. Las respuestas derivadas de las entrevistas realizadas dirigían las respuestas de estas preguntas hacia diversos temas: en el momento de expansión social de las TIC era imposible no hacer una mínima incursión en las mismas, pero la velocidad e incorporación requerida no era compatible con el 
concepto de bienestar subjetivo de la persona (traducido en inquietudes por las sensaciones de no sentirse capaz de utilizar los medios y traducirlos en modos eficaces de desarrollar su docencia).

Además, lo fundamental no es la integración de TIC per sé, sino la adaptación de los procedimientos educativos a las necesidades de los grupos de población más jóvenes. Por tanto, factores emocionales y de formación docente aparecen en el fondo de las respuestas.

Pero se hace necesaria, desde un punto de vista socioeducativo, una de las razones que Area (2014) esgrime para la alfabetización digital en la escuela es

"(...) quien no sepa desenvolverse de forma exitosa con estas herramientas y con las formas culturales que las acompañan inevitablemente tendrá numerosas dificultades para socializarse adecuadamente en la sociedad del siglo XXI."

(Area, 2014: 10)

Los otros argumentos que Area (2014) defiende se relacionan con dos ideas fundamentales: es necesario alfabetizar digitalmente en la escuela porque estamos inmersos en la era digital; y la cultura impresa experimenta un acelerado retroceso en pro de la cultura digital. Es el modo en que la sociedad accede al conocimiento en la actualidad. Es por ello que pretendemos analizar el entorno formativo en el que nos movemos, profundizando a través de este estudio de caso en el análisis de nuestra realidad.

\section{EL ESTUDIO DE CASO: CENTRO DE MAGISTERIO "VIRGEN DE EUROPA" (CENTRO ADSCRITO A LA UNIVERSIDAD DE CÁDIZ)}

La universidad, en el momento de cambio en el que se encuentra por la reciente implantación de los estudios de Grado (Plan Bolonia), constituye un marco privilegiado para incorporar elementos que respondan a ese papel de transformación social que tiene universidad como institución. El sistema de enseñanza lo concebimos como una construcción histórico-social en la que confluyen currículo, profesorado y alumnado (Prendes, 2004) con un acentuado carácter dinámico. Las circunstancias son idóneas para adaptarnos a otros planteamientos metodológicos, ofreciendo una formación inicial y de calidad que responda a las exigencias y que se traduzca en estructuras y modos de organización escolar, métodos de enseñanza y de evaluación... entendidas como innovaciones pedagógicas (Area, 2005). Pero żen qué momento concreto nos encontramos? 
¿Se han introducido las tic en la formación inicial de docentes? estudio de caso en el centro de magisterio "Virgen de Europa"

Las reflexiones provenientes de diversos sectores (en octubre de 2012 se celebraban para analizar el impacto del plan Escuela TIC 2.0 en Andalucía las jornadas Las TIC en la educación de Andalucía: presente y futuro) recogían aspectos como:

- Las carencias normativas existentes en aquel momento en torno al tema.

- La necesidad de señalar las dimensiones de la competencia digital a trabajar, con sus indicadores de evaluación en las diferentes etapas educativas (queremos extender aquí el contexto universitario, aunque no era el objetivo de las jornadas).

- La consideración de la obligatoriedad de incorporación de las TIC a todo el profesorado.

- El reconocimiento específico del valor de la creación de materiales y recursos y publicarlos (implica favorecer competencias en el futuro profesorado que lo posibilite).

- La gran dotación material que se ha realizado en los centros en muy poco tiempo cuando los modelos de enseñanza no siempre responden a las a tales recursos.

- Se aboga por la lógica de la "formación primero-dotación después", tan defendida desde muchos sectores prácticos.

Como puede apreciarse existen una serie de parámetros no definidos fundamentales para que podamos considerar la implantación de las TIC como definitiva, y algunos de ellos van a estar directamente relacionados con la formación del futuro profesorado, en nuestro caso de infantil y primaria.

Aunque la visión es eminentemente negativa. Muchos estudios se han basado en el qué falta y nosotros pretendemos ver qué tenemos ya. Consideramos que los docentes ya hacen esfuerzos significativos en los distintos niveles educativos y la toma de conciencia de este aspecto redundará en una visión más positiva de las posibilidades personales en relación con las tecnologías.

\section{OBJETIVO E HIPÓTESIS}

El objetivo que nos planteamos en este artículo es determinar si se han incorporado y en qué aspectos las tecnologías a la docencia universitaria (con el propósito último de incidir en el diseño de situaciones más adecuadas, partiendo de las áreas de aplicación y mejorando las deficitarias). 
Nuestras ideas de partida contemplaban una serie de hipótesis que recogemos a continuación y que se basaban en el análisis bibliográfico y la propia experiencia:

- Existe una mayor incorporación efectiva de las TIC de la que realmente somos conscientes.

- La incorporación personal depende en gran medida de la vivencia personal con las mismas a lo largo de la trayectoria educativa (obligatoria, postobligatoria) y vital.

- Los alumnos más usuarios de TIC prevén un mayor uso futuro.

- Habrá más conciencia de uso futuro en la fase II que en la I del estudio (probablemente por la difusión de las tecnologías y, en particular, el impacto de las redes sociales).

- Existe conciencia en el Equipo Directivo del Centro de Magisterio "Virgen de Europa" de la necesidad de aplicación de tecnologías a la docencia.

- Existe conciencia entre el profesorado universitario de la necesidad de aplicación a su docencia.

- El uso de TIC no implica grandes modificaciones, entendidas como cambios de paradigmas, en lo que a modelos educativos se refiere.

\section{INSTRUMENTOS DE RECOGIDA DE INFORMACIÓN}

Se ha optado por el diseño de una metodología mixta (cuantitativa-cualitativa) de investigación. Presentamos a continuación las herramientas utilizadas para la recogida de datos.

Cuestionarios:

A. Dirigido a los docentes universitarios (curso 2008-2009). En primer lugar recogemos datos personales (sexo, edad, formación académica, experiencia docente universitaria...), sobre el uso personal de las TIC (frecuencia, herramientas, áreas...), para continuar con una serie de ítems (51) relativos a la experiencia con las tecnologías como profesor de magisterio. Se administró a los 25 docentes del centro y se elaboró en torno a las siguientes áreas:

- Datos personales generales (formación académica inicial, años dedicados a la docencia, uso o no de TIC en diversos contextos...)

- Actitud docente hacia la incorporación: motivos incorporación, momento, ejemplificación... 
- Evaluación actitudes y destreza del alumnado de magisterio: percepciones sobre incorporación en el alumnado, incidencia en diversos niveles...

- Orientación y dinamización de los aprendizajes en el alumnado de magisterio: el papel del profesor en el aula. Influencia en los aprendizajes y la organización del aula.

- Relaciones del profesorado con otros: ya sea profesores y alumnos, profesor-profesor (inter-intracentro)

- Dificultades personales encontradas en la incorporación a su práctica profesional.

B. Dirigido a los alumnos universitarios. El objetivo era conocer la percepción que tienen los futuros profesionales de la educación (actuales estudiantes universitarios) sobre la integración de las TIC en los diferentes contextos formativos por los que ha transcurrido su itinerario educativo, uno de ellos el universitario (el que nos interesa en este artículo). Nos parece relevante en la medida en que son parte integrante de la sociedad del conocimiento y de las tecnologías y se encuentran en la encrucijada de lo que era la enseñanza antes y lo que es en la actualidad. Además, se ven inmersos en el proceso de cambios derivados de la integración del EEES. Por último, la vivencia que tengan de la relevancia, utilidad y posibilidades de la TIC determinarán el uso futuro que hagan de las mismas.

El título del cuestionario es Percepción del alumnado de Magisterio del rol que desempeña el profesor en el ámbito TIC. En primer lugar, solicitamos una serie de datos personales (sexo, edad, curso, especialidad, lugar de residencia...). La escala relativa a la experiencia como estudiante de magisterio de articula en torno a los siguientes apartados:

- Uso docente.

- En mi experiencia como estudiante....

- Influencia en mi manera de aprender

- El uso de las TIC por los profesores...

- Y se concreta en: (pregunta de respuesta abierta)

Entrevistas:

A. La entrevista al Equipo Directivo del Centro de Magisterio pretende:

Definir las motivaciones de la incorporación de las TIC al funcionamiento del centro desde un punto de vista organizativo. 
- Hacer un seguimiento del proceso de incorporación de las TIC (comenzado con los cuestionarios administrados al profesorado en el curso 2008-2009).

- Completar la visión de alumnos y profesorado con la del equipo directivo como promotor de la implantación y estimulación del uso de TIC.

Sacar conclusiones relativas a la importancia de las TIC en la formación de futuros docentes (perfil profesional)

B. La entrevista a profesorado universitario pretende:

- Definir las motivaciones de la incorporación de las TIC al funcionamiento del centro desde un punto de vista docente.

- Hacer un seguimiento del proceso de incorporación de las mismas (comenzado con los cuestionarios pasados al profesorado en el curso 2008-2009).

- Describir las dificultades percibidas en el proceso de incorporación de las tecnologías.

Completar la definición del futuro rol del docente usuario de TIC (actualmente alumnado nuestro) desde la vivencia de la incorporación de las mismas en la práctica universitaria.

- Extraer conclusiones relativas a la importancia de las tecnologías en la formación de futuros docentes

La secuencia conceptual que traduce las entrevistas, se plasma en el siguiente guión:

- motivación para el uso - actitud general y específica del profesor hacia el uso de las tecnologías en el marco del EEES.

- usos concretos, tanto en el área personal como docente.

- ventajas de la incorporación (haciendo referencia a áreas como recursos que posibilita, efecto en la comunicación, participación del alumno, calidad de los aprendizajes...)

- dificultades en el uso (en su práctica concreta, qué dificultades percibe como más destacadas).

En el caso de la entrevista al Equipo Directivo, la visión se completa con preguntas que requieren información sobre:

- Integración de las TIC en el centro, motivos y proceso.

- Importancia concedida

- Efectos en profesores y alumnos.

Se puede observar cómo se pretende hacer una recogida de datos que contemple la visión de los diferentes integrantes de la 
¿Se han introducido las tic en la formación inicial de docentes? estudio de caso en el centro de magisterio "Virgen de Europa"

comunidad educativa mencionada, elaborando instrumentos dirigidos a profesorado, equipo directivo y alumnado.

\section{TEMPORALIZACIÓN}

El cuestionario al alumnado se administra en dos fases:

- la primera de ellas, en el momento que constituye el final de los estudios de magisterio organizados en torno a la diplomatura. En el curso 2008-2009 una muestra de 221 alumnos y alumnas responde a nuestro cuestionario. En ese mismo momento, se administra un cuestionario paralelo al profesorado del centro de magisterio: se empezaban a incorporar las TIC de un modo más sistemático a la metodología docente. Coincide con el periodo de inversión económica más fuerte en el centro en material informático.

- en una segunda fase, con los estudios de grado ya implantados (en el marco del EEES), y el difusión de la tecnología más extendidas a todos los niveles, decidimos administrar de nuevo el cuestionario al alumnado: con una mínimas variaciones respecto a los anteriores (justificadas por necesidades percibidas en el análisis estadístico de los cuestionarios de la primera fase) los alumnos y profesores vuelven a contestar, ahora desde una experiencia mucho más amplia con TIC. La comparación de los resultados de los cuestionarios de la fase I y de la fase II del alumnado de magisterio, nos pareció interesante. Y también nos atrajo profundizar en la percepción del profesorado y del equipo directivo del centro (docentes a su vez) sobre su utilización de las tecnologías en el centro.

\section{RESULTADOS Y DISCUSIÓN}

De las revisiones bibliográficas realizadas concluimos que las buenas prácticas en la utilización de las tecnologías se pueden organizar en torno a cuatro áreas:

- Las relacionadas con los docentes y su formación (fomentan indirectamente relaciones intracentro fundamentalmente, e intercentro en algunos casos...): es necesario formar para usar los recursos que se ponen a disposición del profesorado.

- Las relacionadas con la integración de las TIC en el funcionamiento y organización de los centros (gestión). 
- Las relacionadas con los alumnos (actividades, búsquedas de información, diseño de actividades y materias, estimulación de la motivación, establecimiento de contacto...): el profesorado incorpora las TIC en sus dinámicas cotidianas. El docente, con el uso de tecnologías, aumenta sus posibilidades y capacidad para actuar en el marco del aula, obteniendo recursos útiles para su desempeño profesional, además de resultar un instrumento motivador para el alumnado de la educación obligatoria.

- Las que tienen una repercusión directa en los padres (ya sean de mantenimiento de contacto con los mismos, implicarles en la formación de sus hijos, etc...). Este elemento es poco relevante en el contexto que nos ocupa, al tratarse de alumnado mayor de edad.

Analicemos los resultados de las tres primeras.

Relacionadas con el profesorado

- En el curso 2008-2009: cuestionario revela que, efectivamente, incorporaban mayoritariamente las TIC a su práctica. El resultado era especialmente llamativo en lo que se refería al desarrollo de procesos comunicativos inter e intracentro (ya se tratara de contacto con profesorado o con el alumnado), contexto en el que estaba plenamente difundido el uso. Consideraban como motivaciones fundamentales para la integración el momento socio-histórico y las ventajas que proporcionaba el uso en cuanto a la facilitación de la labor docente. Describían dificultades de tiempo de dedicación al diseño de materiales o asignaturas (o revisión de material digital) 0 de exigencias de formación constantes. El centro y los compañeros suponían un apoyo.

- En el curso 2012-2013, las tecnologías se encuentran plenamente integradas según profesorado y alumnado. Como en el caso anterior, el profesorado se describe como usuario de TIC. La motivación por su uso surge ya sea de modo natural (por la difusión a todos los niveles) que lleva a la incorporación consecuente a la docencia (además facilita la labor docente) o por considerar que formar mediante y en el uso de TIC es una necesidad detectada para el futuro maestro. Tanto en un caso como en otro, las dificultades fundamentales que se encuentra el profesorado universitario tienen que ver con la disposición de tiempo para elaboración y revisión de material digital. El apoyo del Equipo Directivo y los compañeros es una constante. Destacamos la necesidad de formación personal percibida. El profesorado detecta lagunas en su propia formación para la 
incorporación efectiva (fundamentalmente en algunas áreas, como la evaluación). Excepto en casos muy puntuales, los profesores universitarios se formaron inicialmente en un momento en que las TIC no tenían aún el empuje que tienen en la actualidad: eso les ha obligado al autoaprendizaje, a la búsqueda de ayuda de los compañeros (en algunos casos) o a la participación en cursos de formación permanente promovidos por el Centro de Magisterio.

Por otro lado, encontramos en el profesorado entrevistado algunas actitudes importantes para la integración caracterizadas por:

- consideran la educación como un proceso dinámico de adaptación a las necesidades sociales que van surgiendo,

- poseen una imagen del docente universitario como transformador de la realidad social,

- son usuarios de TIC pese a que no les conceden a las mismas más que el valor de recurso material que debe ser rentabilizado con una metodología activa que fomente el aprendizaje del alumnado,

- conciben la docencia como un proceso de actualización constante,

- consideran que las tecnologías deben integrarse plenamente en la docencia en los niveles obligatorios de educación.

De las entrevistas al profesorado del centro de Magisterio podemos concretar que la concepción del proceso de enseñanza-aprendizaje es fundamental y sobre esta, se describe un rol de docente TIC como gestor, mediador y facilitador, entre alumnado/ contenidos y modos de aprendizaje (ver tabla 1). En este contexto debe ser usuario y conocedor del ordenador como medio de organización del trabajo docente (que ayuda a la preparación de documentación personal, apoyos docentes, estimula el trabajo colaborativo, contribuye al diseño de la acción docente, proporciona modos de comunicación efectiva con el alumnado...). Implica ser conocedor de esos códigos de información y comunicación y tener capacidad de seleccionar recursos y de diseñar y producir medios.

Todos los datos aportados confluyen en la delimitación de un rol del alumnado que se caracteriza por:

- Ser más autónomo.

- Con capacidad para valorar la información, seleccionarla, estructurarla y tomar decisiones. 
- Que incorpora la información a los conocimientos previos de modo crítico y personal.

- Defensor del trabajo cooperativo.

- Que busca de modo constructivista la información: descubrimiento de contenidos y el desarrollo de habilidades para la resolución de problemas.

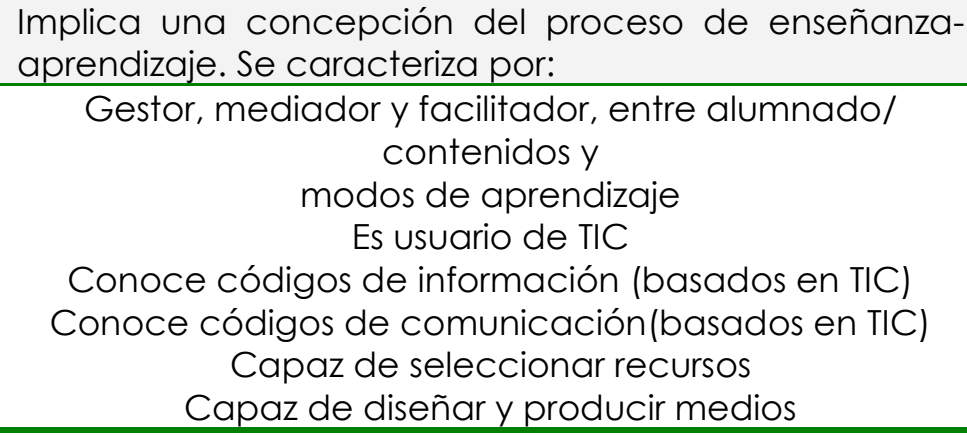

Tabla $n^{\circ}$ 1. Características del rol de docente TIC según el profesorado universitario

\section{Relacionadas con el Equipo Directivo (gestión-administración)}

Las entrevistas al Equipo Directivo se realizan en el curso 20132014. Sitúan el origen de la incorporación en una crisis que se produce en el centro (determinada por una gran bajada de alumnos) y un cambio de dirección. Entre otras actuaciones que no son relevantes para nuestro estudio, la apuesta por la incorporación de las TIC se describe como iniciativa de actuación. En más de una entrevista se hace referencia a la "visión" de la dirección por apostar por ello (no siendo en ese momento las condiciones económicas las idóneas para afrontar la inversión que se hizo).

El apoyo del equipo directivo en su conjunto al desarrollo de metodologías que integren las TIC es incondicional. Como en el caso anterior, se considera que las tecnologías constituyen recursos valiosos que facilitan la labor docente y la gestión del centro, pero que deben estar acompañadas por fundamentos pedagógicos acordes (y/o administrativos, cuando de funciones no docentes se habla). De nuevo, el factor relacionado con la vivencia personal sobre el tema aparece en escena.

\section{Relacionadas con el alumnado}

En ese marco decidimos explorar en qué medida el alumnado del Centro de Magisterio "Virgen de Europa" había sido objeto de integración de TIC en los niveles educativos de enseñanza obligatoria (concretamente en su etapa previa a la entrada a la universidad). Los resultados indican que ni en la fase I (curso 2008-2009) ni en la II (curso 
2012-2013) del estudio, el alumnado percibe haber utilizado de modo sistemático las TIC en bachillerato. Eso sí, en la segunda fase del estudio, más difundido el uso de tecnología y la denominación de centros TIC, el porcentaje de percepción de la incorporación es mayor que en la primera.

En la escala del cuestionario que pretende analizar si perciben el uso sistemático en su etapa de estudiantes de magisterio, de nuevo en ambas fases, encontramos resultados similares, ahora positivos y más acusados en la segunda que en la primera fase del estudio. En esta etapa de su trayectoria educativa parece que las TIC han entrado a integrarse en su proceso formativo, se reconoce explícitamente y es valorado positivamente. Por tanto, podemos considerar que el alumnado va tomando conciencia de la incorporación de las potencialidades de las tecnologías aplicadas al campo docente. Están vivenciando la utilización de las mismas en el contexto del aula de magisterio.

Se percibe al docente que incorpora las TIC es un profesional satisfecho con su profesión. Además consideran que es necesario utilizarlas en su proceso de enseñanza-aprendizaje y que su uso se traduce:

- En lo que se refiere a la comunicación: son clases más participativas, con más contacto entre profesorado y alumnado. Permiten acceder al profesorado más fácilmente.

- En esas clases el alumnado comprende y trabaja mejor los contenidos, al encontrar relación entre realidad y teoría, la posibilidad de ampliar contenidos... lo que se traduce en un mejor aprendizaje.

- La evaluación es más participativa: pese a que el alumnado es consciente de que aquí es donde hay más camino por recorrer.

Si traducimos estos resultados a un rol encontramos a un profesional comprometido con su profesión y satisfecho con la misma, que busca un aprendizaje efectivo en sus alumnos y alumnas ly lo plasma en la elaboración de materiales y el trabajo mediante la plataforma educativa) con predisposición a comunicarse con todos los sectores de la comunidad educativa y dispuesto a reconocer la aportación del alumnado a su propia evaluación. 


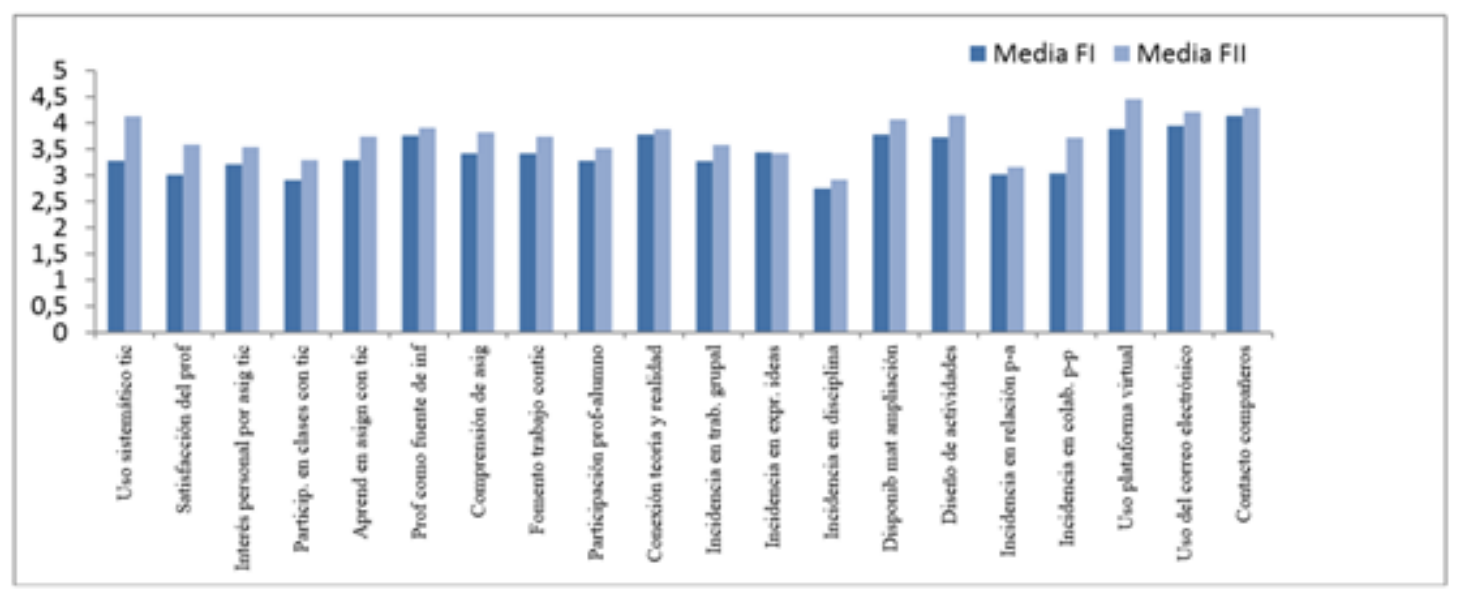

Tabla n². Comparativa de puntuaciones entre las Fases I y II del estudio.

Al analizar la relación entre vivencia personal con las TIC en la trayectoria vital y previsión de uso futuro, resulta muy llamativo comprobar cómo pese a que no han vivido la integración en su vida académica en la educación obligatoria, aunque sí en su etapa universitaria y las tecnologías están plenamente integradas en su vida cotidiana, consideran que las utilizarán. Muy especialmente en la segunda fase del estudio. El alumnado es consciente de la necesidad y las ventajas de la incorporación.

¿Qué se puede concluir de esto en relación con las necesidades formativas percibidas? El perfil del maestro que buscamos en nuestro alumnado sigue siendo el mismo de siempre pero mediado por los recursos que existen en la actualidad y que el alumnado va a integrar en su futura praxis.

Uno de los problemas fundamentales parece provenir del uso que se hace externamente a las instituciones educativas de las tecnologías. Pese a que el uso está tan extendido, el alumnado no domina otras posibilidades de las tecnologías y, además, al haberlas usado en un contexto lúdico-social con fines distintos a los perseguidos en el contexto formativo, nos encontramos con alumnos sin habilidades digitales en los contextos en los que nos interesa. No han vivido en su itinerario formativo obligatorio la integración de las tecnologías ni el desarrollo de competencias digitales. Esa es una las asignaturas pendientes que nos encontramos. Como aspecto positivo, les resulta sencillo integrar destrezas en este campo, al estar familiarizados con las herramientas. Por otra parte, tanto profesorado como alumnado reconocen la gran dificultad que supone incorporarlas a las prácticas evaluativas.

Además, el plan de estudios de nuestro centro (en consonancia con la universidad a la que pertenecemos) no recoge asignaturas que impliquen explícitamente el aprendizaje del manejo de $\mathrm{TIC}$, ya sea con 
fines de usuario o pedagógico, por lo que la utilización queda a libertad del centro educativo y la voluntad de los profesores. Sí se asume implícitamente el uso. De hecho, la inversión en equipos informáticos es alta y constante, además del diseño de cursos de formación en relación con su utilización (ya sean destinados al alumnado del centro o al profesorado).

La necesidad de dedicar tiempo a la revisión del material digital, así como a su preparación son dos de los problemas que se presentan en el profesorado de magisterio y que se recogen explícitamente en las entrevistas. Son además factores coincidentes con los profesionales de la educación de otros niveles educativos (según se concluye del estudio I+D recogido al principio del artículo).

Respecto al proceso de incorporación en el centro se concluye:

- El inicio del empuje de la incorporación en el curso 2008-2009 se ha mantenido y reforzado con los planteamientos docentes y la incorporación de recursos tecnológicos que se encuentran presentes en nuestro centro en cada aula y dependencia administrativa. La plataforma educativa es utilizada por todo el profesorado e independientemente de la mayor 0 menor efectividad de su uso, el alumnado es consciente de la utilización y así lo manifiesta. Se sienten satisfechos con este uso y el profesorado se encuentra en un proceso continuo de formación impulsado desde el propio centro.

- Se completa la visión de alumnos y profesorado con la del equipo directivo como promotor de la implantación y estimulación del uso de TIC: el equipo directivo apuesta por las tecnologías como recurso, sin olvidar la importancia de elementos metodológicos. Conlleva este proceso de explicitación una autorreflexión constante y evaluación de las asignaturas, competencias y resultados de aprendizaje del alumnado. En este marco las herramientas tecnológicas facilitan enormemente la labor al profesorado, así como las funciones administrativas y de gestión del centro.

Y en relación con las hipótesis de partida podemos decir:

- Existe una mayor incorporación efectiva de las TIC de la que realmente somos conscientes. Esta hipótesis se cumple, sobre todo si hacemos referencia al contexto universitario, donde parece que las tecnologías, en nuestro centro, han irrumpido con fuerza. No debemos olvidar que la experiencia personal con las 
tecnologías va ampliándose con lo que la previsión de uso futuro depende en gran medida de la disponibilidad de recursos.

- La incorporación personal de las TIC depende en gran medida de la vivencia personal de las mismas a lo largo de la trayectoria educativa (obligatoria, postobligatoria) y vital. La trayectoria vital parece relevante, así como la trayectoria educativa, pero más en el sentido de que hace al alumnado consciente de las ventajas que las tecnologías pueden ofrecer a la práctica profesional futura. Asimismo, se aprecia que el profesorado de usuario de TIC es más proclive a la utilización de las mismas en su práctica laboral.

- Los alumnos más usuarios de TIC prevén un mayor uso futuro. No hemos podido establecer una correlación positiva entre estos dos factores. Un gran porcentaje prevé un uso futuro y se declaran como usuarios de TIC pero muy pocos han las utilizado sistemáticamente en su formación obligatoria.

- Habrá más conciencia de uso futuro en la fase Il que en la I del estudio (probablemente por la difusión de las tecnologías y, en particular, el impacto de las redes sociales). Sí que encontramos resultados más positivos en todos los ítems en la escala que prevé la utilización de TIC en el futuro en la fase II respecto a la I. No podemos comprobar el origen de tal tendencia podría decirse que la difusión de las tecnologías en general y de las redes sociales en particular tenga alguna relevancia en este hecho.

- Existe conciencia en el Equipo Directivo del Centro de Magisterio "Virgen de Europa" de la necesidad de aplicación de TIC a la docencia. Existe una clara conciencia de la relevancia de la incorporación, que se traduce en una amplia inversión inicial y en la continuidad de las mismas en el centro.

- Existe conciencia entre el profesorado universitario de la necesidad de aplicación de TIC a su docencia. Efectivamente, existe tal conciencia. Se utilizan en mayor o menor grado en la docencia, con mayor o menor efectividad, pero llama la atención como afecta al $100 \%$ del profesorado en cuanto al fomento de los canales de comunicación mediante correo electrónico. El profesorado es consciente de las ventajas que aportan las tecnologías, aún cuando perciban que les quedan elementos por incorporar. 
- El uso de TIC no implica grandes modificaciones, entendidas como cambios de paradigmas, en lo que a modelos educativos se refiere. Consideramos que efectivamente se corrobora la hipótesis. El profesorado usuario de TIC tiene unas características que llevan años gestándose y que se originan en la propia inquietud docente de responder a las necesidades sociales. Es por ello que consideramos que la actitud de base es la misma.

\section{CONCLUSIONES}

Queremos concluir con una serie de reflexiones que aúnan resultados encontrados y teoría analizada en la primera parte de nuestra investigación.

\section{Sobre la cultura de la enseñanza.}

M. Foucault (Popkewitz, 1987) sostiene que en cualquier momento de la historia, la sociedad está mantenida por unos códigos fundamentales de cultura que gobiernan el discurso de dicha sociedad en todos los campos, desde los políticos a los técnicos, desde los científicos a los pedagógicos... Dichos códigos, se terminan convirtiendo en régimen de verdad y conforman y definen qué es aceptable y qué no lo es, qué es verdad y qué es un error y varían en función de las idiosincrasias políticas o sociales.

Foucault propone unos elementos en la vida social fundamentalmente influyentes:

- El primero de ellos hace referencia a la profesionalización del conocimiento. Considera que para controlar los elementos de la vida institucional, se han creado ocupaciones: el poder se ejerce mediante la producción y la reproducción del conocimiento creado vigente. De este modo, el conocimiento socioprofesional debe organizar la vida social y privada.

- El segundo de ellos, hace referencia al conjunto de tensiones sociales y culturales que determinan la compleja situación de la escuela.

Cuando estos dos elementos interactúan ser producen los denominados códigos de la cultura de la enseñanza lque determinarían las características destacadas por las personas en un contexto determinado que desembocarían en sus modos de actuación). 
En definitiva, el problema de la educación y de la formación estriba en identificar los medios más apropiados para la consecución de los fines establecidos y en aceptar que pueden modificarse medios anteriormente aceptados. A través de este argumento podemos justificar el momento por el que ha atravesado la educación en lo que a la incorporación de cambios se refiere, incluido el que hace referencia a las tecnologías.

Si consideramos que la educación es el registro, revisión, gestión, expresión, formación, adiestramiento y comprensión del pensamiento humano con el objetivo de transmitir el saber y la memoria de cada civilización, y al mismo tiempo, reinventar este marco en cada generación y proporcionar las herramientas para repensarlo, la necesidad de avanzar es innegable (Perceval y Tejedor, 2008) y además es un proceso no concluido.

"...deberíamos reorientar los objetivos en función de la cultura circundante, así s procedimientos y técnicas. Necesitamos cambiar la manera de trabajar, tanto individual como grupalmente, nuestra relación con la organización del centro y la forma de acceder a la información...".

(Pavón, 2001: 42)

Por otro lado, centrándonos en el aprendizaje del alumnado, y partiendo de la aportación de Pastor Alcaide (2013), que a su vez se basa en clásicos del aprendizaje social como Bandura, los alumnos parten de zonas de desarrollo próximo diferentes hoy en día, se han ampliado con las tecnologías y las redes sociales. Ya no necesitan al adulto o experto presente necesaria y continuamente para ampliar 0 conseguir los aprendizajes sino que hay entornos extra en los que el alumno puede acceder a información. Pese a las dificultades que indudablemente plantea su uso, estos entornos se constituyen en lugares de oportunidad para la mejora de procesos tradicionales de enseñanza-aprendizaje, como también comenta Marqués-Graells (2013), cuando propone la introducción de nuevas tecnologías docentes para contribuir a la mejora de la formación y los resultados académicos de los estudiantes y lo explica mediante el modelo TPACK (Marqués-Graells, 2013). En este contexto, los profesionales de la educación se van adaptando a estos contextos, y lo hacen de un modo más efectivo y frecuente del que es oficialmente reconocido quizá. 
¿Se han introducido las tic en la formación inicial de docentes? estudio de caso en el centro de magisterio "Virgen de Europa"

\section{Sobre la figura del docente.}

Nos preguntábamos si ser maestro hoy es lo mismo que ser maestro en tiempos anteriores y estamos de acuerdo con Canelles, J. (2013) en su consideración de que no parece haber gran diferencia. Así la sociedad de la información y del conocimiento define un entorno que requiere otros modelos de enseñanza-aprendizaje o adaptación de lo previos de modo efectivo. En la medida en que los adelantos técnicos ponen a disposición de los profesionales de la educación una batería de herramientas que facilita tal adaptación, podemos considerar que el maestro con el uso de TIC aumenta sus posibilidades y capacidad para actuar. Por tanto, dejar de hacerlo implica no ser un profesional competente. Hablamos de un perfil que requiere unas actitudes de partida en un marco de un respeto a la diferencia que nos caracterice a cada uno y que enriquece la realidad con la diversidad personal.

Estas actitudes se concretan en roles de GESTORES de formación. El docente se define en base a la mediación y la facilitación de aprendizaje entre alumnos y modos de aprendizaje/contenidos concretos. El desarrollo de tal perfil se imbrica en una red de relaciones en las que influimos y nos vemos influidos por el rol del propio alumno, en el que buscamos otros elementos (fundamentados en el aprender a aprender y el fomento de la autonomía de los aprendizajes). Como en el caso del rol docente, el rol del alumno tampoco varía tanto: el ser humano se caracteriza por su capacidad y deseo de aprendizaje y esto se han producido en todas las épocas, con la mediación del tutor o sin ella. Lo que se modifican son las especificaciones teóricas sobre lo que esperamos y no el fondo, los recursos de los que disponemos y no el hecho en sí. Seguimos queriendo aprender y buscamos tal aprendizaje: antes en los contextos de referencia y ahora también. La característica permanece, el contexto varía así como los recursos.

Como recogen Solé, Colom y Arana (2013), las experiencias de la vida profesional de maestros y maestras son fundamentales en el desarrollo de determinados elementos profesionales. Y eso es lo que hemos constatado. Son esas experiencias, diversas y personales, las que determinan opciones en determinados momentos por un camino $u$ otro. Al analizar lo que el profesorado nos cuenta no analizamos la realidad, sino cómo la vivió singularmente, condicionada por unas circunstancias únicas del centro donde la desarrolló y por las personas que integran el contexto en cuestión (identidad institucional y cultural del contexto específico más inmediato). 
Por otro lado, el impacto de las tecnologías es sorprendente. Y uno de los campos es el desarrollo de las redes sociales virtuales: Colás, González y de Pablos (2013) recogen cómo el estudio de las mismas es un objetivo de creciente estudio en los últimos años y un elemento fundamental de la difusión de las TIC. Internet se ha convertido en una herramienta de diálogo y comunicación, básica en el entretenimiento y en el disfrute del tiempo de ocio.

Paralelamente, y puede que por la velocidad de los cambios, la relación entre los medios y la educación ha sido durante mucho tiempo foco de muchas esperanzas y temores, como recogen Buckigham y Martínez-Rodríguez (2013). En este sentido se ha temido por la figura docente aún cuando

"Para el profesorado tanto los centros como los profesores innovadores no son fruto de la integración de las TIC, sino que ambos lo eran antes de la llegada de las TIC; las TIC le han permitido potenciar su innovación mediante herramientas que posibilitan nuevas acciones $u$ optimizar las que realizaban antes, pero su modelo educativo no ha cambiado sustantivamente".

(Ortiz, Peñaherrera y Ortega, 2012:12)

Según Perceval y Tejedor (2008) se constata cierto temor por parte de algunos sectores de profesorado ante una posible sustitución de la figura docente por la máquina. Pero es un miedo que no se corrobora con los resultados de nuestro estudio: en todos los casos, el docente sigue siendo una figura fundamental en el aula, sigue siendo incluso depositario de la información fundamental... pero la gestiona de otra manera.

Sobre la formación del docente.

Es en este contexto en el que surgen las necesidades de formación

"(...) el profesor es por encima de todo un intelectual en la encrucijada del conocimiento y de la cultura, un intelectual que reflexiona sobre la ciencia, la cultura y el arte como instrumentos de adaptación creadora a la realidad, con el propósito de provocar su asimilación en las nuevas generaciones".

(Pérez Gómez, 2007:19)

Si partimos de la necesidad de reflexión constante, es una formación en constante reinvención. lón y Cano (2012) desarrollan una serie de entrevistas a nivel universitario en cinco Universidades y 
concluyen claramente que es necesario ofrecer una formación más coherente adecuada a los retos del EEES. Ya estamos en esta línea: el profesorado universitario, consciente de sus limitaciones y lagunas, investiga y se forma, incorpora en su docencia elementos no incluidos previamente, en un intento de responder a la demanda social, entendida como contexto de referencia. Ofrecen, por otro lado, una serie de modelos y parámetros de partida a los futuros docentes, fundamentándose como figuras de referencia para el profesional de la educación del futuro. Ell, Hill y Grudnoff (2012) describen un estudio en el que los conocimientos previos y la experiencia personal juega un papel fundamental en el posterior uso de las TIC. Consideran que estas experiencias influyen decisivamente en el modo de aprender y de enseñar posteriormente de los futuros maestros. La experiencia educativa impregna la conciencia sobre la formación y se traduce en planteamientos determinados (y metodologías): "El desarrollo profesional del profesorado es producto del desarrollo pedagógico que va adquiriendo a lo largo de su vida" (Imbernón, 2013:13).

Y aquí encontramos un matiz en la formación universitaria que no se recoge tanto como se debiera: su responsabilidad en la formación de ciudadanos responsables. Desde este enfoque, Lorenzo (2012), considera la enseñanza universitaria una oportunidad para el desarrollo de las competencias cívico-sociales en el alumnado universitario y la base para asumir cambios en la cultura docente, que sería la base sobre la que construir el conocimiento del futuro (entendiendo futuro como mañana y no como una realidad que siempre está por llegar y que no termina de plasmarse en planteamientos concretos).

Martínez (2012) recoge que en este contexto la consideración de que pese a que la formación en las tecnologías es importante, debemos recordar que el énfasis debe hacerse en la pedagogía y no en la tecnología.

\section{PROSPECTIVA}

Podemos decir, por tanto, que la difusión de la tecnología alcanza todos los niveles formativos, especialmente en el caso del centro que nos ocupa (Centro de Magisterio), observándose una evolución más positiva en los últimos años en lo que a su aplicación y difusión se refiere. En este punto podemos resaltar que las inversiones en tecnología han sido y son una constante en nuestro centro, ampliando los recursos disponibles y proporcionando formación según se van detectando necesidades. Se facilita de este modo el acceso a los 
mismos por la gran mayoría del profesorado universitario, que, al mismo tiempo, ha recibido cursos de formación en este sentido.

En cuanto a los procesos de enseñanza aprendizaje, claramente, el profesorado universitario del Centro de Magisterio "Virgen de Europa" considera que los procesos de enseñanza-aprendizaje se han modificado por el momento histórico en el que vivimos. Incidirá en la preparación de nuestras nuevas generaciones, clientes de los docentes del futuro. Implicará una revisión de los planteamientos metodológicos pero nos puede socorrer a la hora de definir un perfil docente que recupere el prestigio social en base a una autoridad moral, sustentada sobre la profesionalidad.

Por ello, es necesario seguir trabajando en la definición del perfil profesional docente, incidiendo en el desarrollo de capacidades de formación permanente y actitudes favorables al cambio, que redundarán en la formación de alumnos más responsables y capaces. Será un perfil que quizá nunca termine de definirse pues "Ias enseñanzas de la vida y las costumbres del pasado, no son suficientes para afrontar los desafíos del presente y las exigencias del futuro" (Pérez Gómez, 2007: 96)

Este proceso en el que nos encontramos inmersos es previsible que continúe en la misma línea, incluso que alcance proporciones más altas (en lo que a velocidad de cambio se refiere y disponibilidad de información). Y en la medida en que el ser humano siga desarrollando tecnología, otros serán capaces de aplicarlas a los distintos contextos.

Nos reiteramos en un uso de las TIC en el marco de una concepción de la educación activa, participativa en la que estas aparecen como recurso o herramienta con muchas ventajas pero que no son decisivas en los planteamientos didácticos o instructivos del docente, cuyo papel se ha modificado pero por la lógica evolución social y no se considera que vaya a desaparecer su figura en pro de otras formas alternativas de educación que sustituya la figura del docente, sino que ésta simplemente se transformará. Y de que ya estamos inmersos en el proceso.

En esta perspectiva optimista del desarrollo social y docente, la perspectiva formativa del profesorado también se hace eco de los requerimientos sociales: la universidad responde al papel transformador que tiene a nivel social y señala el camino del desarrollo formativo constante partiendo de la actitud de sus propios docentes. Sería muy interesante poder contactar con nuestro alumnado cuando se encuentre desarrollando su labor profesional y analizar el grado y la 
¿Se han introducido las tic en la formación inicial de docentes? estudio de caso en el centro de magisterio "Virgen de Europa"

medida en que incorporan las tecnologías en su docencia. Y más interesante aún: cuáles serán esas tecnologías que emplean.

\section{REFERENCIAS BIBLIOGRÁFICAS}

AREA, M. (2014). Alfabetización digital y competencias profesionales para la información y la comunicación. Organización y Gestión Educativa, $\mathrm{n}^{\circ}$ 1, pp. 9-13.

CANELLES, J. (2013). Hacer de maestro hoy. Aula de innovación educativa, 218, 29-33.

ELL, F., HILL, M. Y GRUDNOFF , L. (2012). Finding out more about teacher candidates' prior knowledge: implications for teacher educators. Asia-Pacific Journal of Teacher Education. 40 (1), 55-65.

DOI: http://dx.doi.org/10.1080/1359866X.2011.643760

IMBERNÓN, F. (2013). Incidencia de la formación en el desarrollo y ejercicio de la profesión docente. Aula de innovación educativa, 218, 25-28.

ION, G. Y CANO, E. (2012). La formación del profesorado Universitario para la implementación de la evaluación por competencias. Educación XXI, 15.2, 249-270

DOI: http://dx.doi.org/10.5944/educxx1.15.2.141

LORENZO, M. (2012). La función social de la universidad y la formación del profesorado. EDETANIA, 42, 25-38.

MARQUÉS-GRAELLS, P. (2013). Nuevas metodologías docentes. Aula de Innovación, 218, 16-21.

MARTíNEZ, J. (2012). Impact of the proliferation of information and technology in Higher Education. Aula Abierta, 40 (3), 97-106.

ORTIZ, A.M., PEÑAHERRERA, M. Y ORTEGA, J. (2012). Percepciones de profesores y estudiantes sobre las TIC. Un estudio de caso. EDUTEC. Revista Electrónica de Tecnología Educativa, 41, Septiembre.

PASTOR ALCAIDE, J.L (2013). Aprendizaje social y nuevas formas de entender el proceso de e-a. Padres y Maestros, 351, 11-15.

PAVÓN RABASCO, F. (2001). Educación con nuevas tecnologías de la información y la comunicación. Sevilla: Kronos.

PÉREZ GÓMEZ, A., MARTíNEZ TEY, A., ESCOMBRA, M.A. Y GONZÁLEZ, M.T (2007). Profesorado y otros profesionales de la educación. MEC. Madrid: Octaedro.

POPKEWITZ, T. (eds)(1987). Formación del profesorado. Tradición. Teoría. Práctica. Valencia: Servicio de Publicaciones de la Universidad de Valencia.

PRENDES, M.P. (coords) (2004). Nuevas tecnologías y educación. Madrid: Prentice Hall. 
SOlÉ, I., COLOM, C. y ARANA, A. (2013). Testimonios de aula. Aula de innovación educativa, 218, 16-21.

\section{FUENTES ELECTRÓNICAS}

AREA, M. (2005). Tecnologías de la información y la comunicación en el sistema escolar. Una revisión de las líneas de investigación. Revista electrónica de Investigación y Evaluación Educativa. Vol. 11, 1. Consultado en Septiembre de 2009 en http://www.uv.es/RELIEVE/V1On2/RELIEVEV11_1.htm

BUCKINGHAM, D. Y MARTÍNEZ-RODRÍGUEZ, J.B. (2013). Jóvenes interactivos: nuevas ciudadanía entre redes sociales y escenarios educativos. Comunicar, 40, XX, 10-13.

DOI: http://dx.doi.org/10.3916/C40-2013-02-00

COLÁS, GONZÁLEZ, T. Y DE PABLOS, J. (2013). Juventud y redes sociales. Motivaciones y usos preferentes. Comunicar, 40, XX, 15-23. Consultado en Julio 2013 en

http://www.revistacomunicar.com/index.php?contenido=revista\&numer o=actual

PERCEVAL, J.M. y TEJEDOR, S. (2008). Los cinco grados de la comunicación en educación. Revista Comunicar, 30, XV, 155-163. DOI: http://dx.doi.org/10.3916/c30-2008-02-009

\section{Sobre la autora:}

Claudia I. Ruiz López

Centro de Magisterio "Virgen de Europa"

Docente Titular del Departamento de Psicología del Centro de Magisterio "Virgen de Europa", adscrito a la Universidad de Cádiz (desde el curso 19951996). Doctorada en Departamento de Didáctica de la Universidad de Cádiz. Tesis: "El rol del docente y las buenas prácticas educativas basadas en el uso de tic: descripción de casos en centros educativos del Campo de Gibraltar" (dirigida por D. Juan de Pablos Pons y D. Juan Casanova Correa). Diciembre, 2014 y Miembro del Grupo de Investigación, Evaluación y Tecnología Educativa (GIETE). Universidad de Sevilla.

\section{Para citar este artículo:}

Ruiz, C. (2015). ¿̇Se han introducido las tic en la formación inicial de docentes? estudio de caso en el centro de magisterio "Virgen de Europa". Revista Fuentes, 16, Junio, pp. 131-154. [Fecha de consulta: $\mathrm{dd} / \mathrm{mm} /$ aada]. http://www.revistafuentes.es/

D.O.I.: http://dx.doi.org/10.12795/revistafuentes.2015.i16.06 
¿Se han introducido las tic en la formación inicial de docentes? estudio de caso en el centro de magisterio "Virgen de Europa" 